\title{
Cumulative Effects of Occupational Loading as a Risk Factor for Hip Pain in UK Military Personnel - Study Protocol of a Case Control Study
}

\section{Russell J Coppack ${ }^{1,2 *}$, James L Bilzon ${ }^{2}$, Andrew K Wills, , Ian M McCurdie ${ }^{4}$, Daniel T Walton ${ }^{2,4}$, Alastair $M$ Nicol $^{4}$ and Alexander $N$ Bennett ${ }^{1,5}$}

\author{
${ }^{1}$ Academic Department of Military Rehabilitation, Defense Medical Rehabilitation Centre (DMRC), UK \\ ${ }^{2}$ Department for Health, University of Bath, UK \\ ${ }^{3}$ School of Clinical Sciences, University of Bristol, UK \\ ${ }^{4}$ Centre for Lower Limb Rehabilitation, UK \\ ${ }^{5}$ Leeds Institute of Rheumatic and Musculoskeletal Medicine, University of Leeds, UK
}

*Corresponding author: Russell J Coppack, Academic Department of Military Rehabilitation, Defense Medical Rehabilitation Centre, Headley Court, Epsom, Surrey, UK, Tel: (+44)-1372947103, E-mail: russ.coppack100@mod.uk

\section{Abstract}

Background: High rates of hip Osteoarthritis $(\mathrm{OA})$ have been reported in occupational sub-groups exposed to prolonged and strenuous physical activity levels. The military population is particularly at risk given the demands inherent to this population. Despite the burden associated with non-arthritic hip complaints and OA there is a dearth of published data investigating the pre-disposing risk factors in military populations. The purpose of this article is to describe the protocol and methodology for a case-control study examining the influence of occupational and demographic factors associated with physician-diagnosed non-arthritic hip pain among active UK military personnel.

Methods/Design: The study design is a population based case-control study. Cases $(n=100)$ will be recruited from patients referred with hip pain to the Defense Medical Rehabilitation Centre (DMRC) Headley Court, UK. Age-matched controls $(n=400)$ will be randomly selected from the entire UK military population. Information on pre-enlistment activity levels, military branch titles, exposure to occupational physical stress, exposure on deployed operations, sports participation and other individual risk factors will be collected and evaluated using a standardized questionnaire. The test-retest reliability and internal consistency of the study questionnaire in a military population will be assessed with a sample of healthy male military permanent staff $(n=50)$ from DMRC Headley Court.

Discussion: This case-control study will provide the first estimates of the risks associated with occupational factors and the onset of non-arthritic hip pain in UK military personnel. If aspects of specific occupational activities and tasks contribute to the occurrence of hip pain it will inform future prevention strategies.
\end{abstract}

\section{Keywords}

Hip pain, Occupational loading, Risk factors, Military personnel

\begin{abstract}
Abbreviations
ADMR: Academic Department of Military Rehabilitation; ANOVA: Analysis of Variance; BMI: Body Mass Index; Cl: Confidence Intervals; DMRC: Defense Medical Rehabilitation Centre; FAl: Femoro Acetabular Impingement; ICMJE: International Committee of Medical Journal Editors; ICC: Intra Class Correlation Coefficients; MIAC: Multidisciplinary Injury Assessment Clinic; MOD: Ministry of Defence; MODREC: Ministry of Defence Research Ethics Committee; MPQ: Military Pre-Training Questionnaire; MPLQ: Military Physical Loading Questionnaire; OA: Osteoarthritis; OR: Odds Ratios; RCT: Randomized Controlled Trial; UK: United Kingdom; US: United States
\end{abstract}

\section{Introduction/Background}

\section{Hip osteoarthritis and non-arthritic hip disorders}

Hip Osteoarthritis (OA) is a prevalent chronic musculoskeletal condition causing pain, disability and reduced quality-of-life in affected individuals [1]. Symptomatic hip $O A$ is a clinical diagnosis derived primarily on pain in the presence of radiographic features including osteophytes, joint space narrowing, subchondral sclerosis, and cysts [2]. There is currently no cure, and total hip joint replacement is common for advanced disease. The prevalence of radiographic and symptomatic hip OA increases with age and is estimated to be $15 \%$ (radio- 
graphic) and 5\% (symptomatic) among Caucasians aged 55 years and over [3]. While progression between onset of hip pain to severe symptoms and end-stage disease is variable, disease progression generally appears to be much more rapid than that observed in knee OA [4]. Thus hip OA is considered to be one of the most serious musculoskeletal disorders from a public health standpoint contributing to substantial patient morbidity, health care costs and lengthy surgical waiting lists [1-4].

In the past decade significant progress has been made in understanding the patho mechanics of hip disorders occurring before the onset of OA. Non-arthritic hip pain lacks a universally accepted definition but refers to a collection of conditions involving intra-articular structures including Femoroacetabular Impingement (FAI), structural instability, labral tears, chondral lesions, and ligamentous teres tears [5]. These conditions are not necessarily mutually exclusive and may be inter-related. FAl is recognized as a common cause of non-arthritic hip pain and is defined as a motion-related clinical disorder of the proximal femur and acetabulum that may result in pain and decreased function $[6,7]$. This impingement is caused by abnormal morphology of the femoral head/neck junction (cam impingement), acetabular rim (pincer impingement) or both [8].

Recent literature reports an estimated prevalence rate of $23 \%-67 \%$ radiographic $\mathrm{FAl}$ in the general population [9]. FAl can lead to labral tears and chondral damage [10], and cam type FAI can increase the risk of developing OA [11]. However, despite the considerable prevalence of FAI morphology, only a proportion of individuals develop symptomatic OA [12] and the specific risk factors including occupational considerations causing non-arthritic hip pain and FAl are unclear.

\section{Occupational mechanical loading as a risk factor for hip pathology}

To better understand the population-based epidemiological characteristics of hip OA, numerous studies have sought to determine the risk factors for this disease. Risk factors for hip OA include a wide range of local and systematic features including age, genetic disposition, previous injury, obesity, and occupation $[3,4]$. Particularly high rates of hip OA have been reported in occupational sub-groups including professional athletes and military personnel [13]. It is widely believed the higher rates of OA seen in these sub-groups is linked to the increased physical activity and greater mechanical loads on the hip over time compared with sedentary occupations $[2,13]$.

Despite the intuitive appeal of an association between higher recreational and sporting activity levels, accelerated joint degradation, and increased risk of hip $\mathrm{OA}$, strong evidence indicating a causal link remains elusive [1-4,13]. There is, however, considerable evidence suggesting an association between occupational lifting and hip OA [14-19]. Two recent population-based studies of Finnish men and women reported a work history of lifting loads greater than $20 \mathrm{~kg}$ revealed a strong association with hip OA in all age groups [14,15]. An earlier Japanese case-control study found a strong association between heavy lifting at work and the risk of OA [16]. In this study, subjects who regularly lifted loads in excess of $25 \mathrm{~kg}$ during their first job (unadjusted OR 3.6, 95\% $\mathrm{Cl}$ 1.3-9.7), and $50 \mathrm{~kg}$ in their main reported job (unadjusted OR 4.0, 95\% Cl 1.1-14.2) were at significantly increased risk of developing hip OA. In general agreement with these findings, three systematic reviews concluded there was moderate to strong evidence for a causal relationship between heavy lifting over a 10-20 year period and hip OA. A twofold increased risk in specific occupational groups including farming, mining, fishing and construction work was reported after 10-years exposure [17-19]. However, the size of the effect estimates and the specific occupational activities reported to increase risk vary between studies. To date, no studies have investigated cumulative exposure to occupational mechanical loading of the hip joint as a risk factor for non-arthritic hip pain and FAI.

\section{Non-arthritic hip pain and $O A$ in the military}

Although OA has traditionally been considered a disease that affects older people, in whom the incidence increases with advancing age, recent studies suggest that the majority of adults with $O A$ are younger than age 65 years [20]. The military population is particularly at risk given the demands inherent to this population [21-25]. However, there is a dearth of published data demonstrating the prevalence of FAl, intra-articular hip pathology or hip OA in a military population.

One U.S. study found a high prevalence (87\%) of radiographic abnormalities suggestive of FAl in a cohort of 155 young army males [21]. Another large U.S. based study reported the incidence rates and epidemiological variables of hip OA in the Armed Forces [22]. In a military Population at Risk (PAR) of 12,096,304 person-years, the overall unadjusted incidence rate of hip OA was 54 cases per 100,000 person years among women, and 32 cases per 100,000 person years among men. Women, when compared with men, had a significantly increased adjusted incidence rate ratio for hip OA of 1.87 (95\% Cl 1.73-2.01). In an earlier case-control study of US Military veterans attending an outpatient clinic to obtain hip radiographs, Roach, et al. [23] found ex-servicemen who had been exposed to heavy lifting for at least 15 years had 2.5 times higher odds of hip OA than men with light work $(95 \% \mathrm{Cl} 1.5-5.0)$; this association was statistically significant $(p<0.0001)$. These authors concluded the biomechanical aspects of lifetime military service contribute to the risk of hip OA.

Two further studies reveal similar trends regarding occupational risk and the development of hip pathology in military personnel $[24,25]$. In both studies the branch 
of military service and military rank were important occupational factors associated with a higher incidence of hip OA.

Recent reviews have concluded there is moderate to strong evidence for heavy lifting and farming as a risk factor for hip OA $[17,26]$. Military service is somewhat comparable to farming and heavy manual labor in that the occupational demands often require regular lifting, prolonged standing, adoption of awkward postures, and carrying heavy loads over rough terrain [22]. Standard training for serving military personnel involves intensive military occupational specialty (e.g. infantry) training, and the physical capacity to perform prolonged combat deployments in austere environments. Furthermore, abnormal femoro acetabular morphology, especially CAM deformity, is commonly seen in the young, active men who make up much of the military population [27]. Many soldiers join the service in their late teens when the hip joint is not fully developed, and the hip may be particularly vulnerable to trauma or physical stress at this stage of life [28]. Consequently, the military population is a high risk for hip disorders given the demographics and demands inherent with this population.

Despite the burden associated with non-arthritic hip complaints and OA the predisposing risk factors in a military cohort have not been reported. We are also unaware of any studies examining the impact of career long cumulative exposures to physical workloads on the risk and timing of hip pathology, at a population level, in Armed Forces personnel. Understanding "how" a risk factor is acting over time is important as it could provide an insight into the aetiology of a condition in the general field of occupational health. Research is also necessary to ensure a currently unutilized potential for prevention is not missed [29].

\section{Methods/Design}

\section{Study aims}

The active duty UK military population provides an excellent opportunity to examine the incidence and risk factors of non-arthritic hip pain in a young, physically active population that is regularly exposed to repetitive joint loading during occupational tasks. However, the lack of recent data makes it impossible to comprehensively describe the current burden of this health problem and place it in its proper context.

The purpose of this study is to examine the influence of occupational and demographic factors associated with physician-diagnosed non-arthritic hip pain among active UK military personnel.

\section{Primary aims}

1. Evaluate the risk of non-arthritic hip pain in relation to lifelong cumulative occupational physical workload in UK military personnel.
2. Evaluate the risk of non-arthritic hip pain in relation to age, rank seniority, and service branch in UK military personnel.

3. Determine the risk associated with specific occupational factors (e.g. load carriage, heavy lifting, endurance marching) and the onset of non-arthritic hip pain in UK military personnel.

\section{Secondary aims}

The secondary aims will be to examine the association between recreational physical activity (e.g. jogging, swimming, cycling), sports participation, and lifestyle factors (e.g. smoking, alcohol consumption), and non-arthritic hip pain in UK military personnel. Because current mechanical loading may be influenced by current symptoms, this study will principally be concerned with cumulative (past) exposures influencing the onset of symptoms [2]. The study also aims to establish if cumulative exposure risk can be accurately measured in a military population as well as piloting a new data collection questionnaire tool.

\section{Primary hypothesis}

Occupational physical activity, particularly heavy lifting, does predispose non-arthritic hip pain in UK military personnel.

\section{Secondary hypothesis}

1. Military rank, seniority and age will be associated with non-arthritic hip pain.

2. Branch of military service (e.g. Navy, Army, Air Force) will be associated with non-arthritic hip pain.

\section{Study design}

The study design is a population based case-control study. To obtain information on military branch titles, exposure to occupational physical stress and other risk factors, the study is nested in a Randomised Control Trial (RCT) of military personnel undergoing hip joint rehabilitation. To date, no studies have compared the effects of Multidisciplinary Team (MDT) residential rehabilitation interventions versus conventional out-patient care for intra-articular hip pain. With no available data the effectiveness of MDT residential care remains unclear. Therefore, we are conducting a randomised control trial to compare the effects of a residential MDT intervention versus usual out-patient treatment on the clinical outcomes of UK Military personnel with hip pain. This trial will be conducted at the UK Defense Medical Rehabilitation Centre (DMRC) Headley Court. The RCT will recruit $\mathrm{n}=100$ participants with a clinical diagnosis of intra-articular hip pain and these participants will be approached to also volunteer as case participants in this case-control study. The protocol design, methodology and analysis plan for the RCT has been published elsewhere [30]. 
In addition to occupational exposures, data on other risk factors for hip pain and OA will be collected and evaluated including age, Body Mass Index (BMI), previous joint disease, and sport and other non-occupational activities that may increase the risk of hip pathology. To balance cost-effectiveness and study quality, use of the nested case-control design is recommended in which cases and controls can be identified within a well-defined baseline population [18].

\section{Inclusion and exclusion criteria of case and control participants}

Cases will be recruited from patients attending the centre for lower-limb rehabilitation injury assessment clinic at the Defense Medical Rehabilitation Centre (DMRC) Headley Court, UK with physician diagnosed symptoms of intra-articular hip pain. Controls will be recruited from across the entire UK military population.

Case participants: Patients with clinical indicators of non-arthritic, intra-articular hip pain meeting the following inclusion/exclusion criteria [31] will be eligible for recruitment as case participants.

\section{Inclusion criteria:}

I. Male.

II. Anterior or lateral hip pain for at least 3-months.

III. Clinical signs and symptoms of non-arthritic intra-articular hip pathology/FAl diagnosed by a specialist Rehabilitation/Rheumatology/Sports and Exercise Medicine Consultant Physician.

IV. Physical examination findings or reproduction of pain in the groin or lateral hip with the log roll, anterior hip impingement test, resisted straight leg-raise test or Thomas test.

V. Aged $\geq 18$ years.

VI. Full-time regular military personnel.

\section{Exclusion criteria:}

I. Female.

II. Inflammatory arthropathy.

III. History of any injury or illness that would limit or prevent exposure to occupational manual labour or physical stress.

IV. Hip infection or tumour.

V. Hip fracture.

VI. Major structural deformity of the hip.

VII. History of congenital/adolescent hip disease.

VIII. Clinical signs of lumbar spine disease including radiculopathy.

IX. Aged $\geq 50$ years.

$X$. Insufficient capacity to provide informed consent.
XI. Part-time reservists (e.g. not full-time, regular military personnel).

Control participants: UK Military personnel meeting the following criteria will be eligible for recruitment as control group participants. Control participants will be matched with each case for age (year of birth).

\section{Inclusion criteria:}

I. UK Armed Forces personnel.

II. Male.

III. Aged $\geq 18$ years.

IV. Full-time regular personnel.

\section{Exclusion criteria:}

I. Female.

II. History of any hip pathology or injury including congenital disease, fracture, OA, FAl or physician diagnosed hip pain lasting $>3$-months.

III. History of any injury or illness that would limit or prevent exposure to occupational manual labor or physical stress.

IV. Insufficient capacity to provide informed consent.

V. Aged $\geq 50$ years.

VI. Part-time reservists (e.g. not full-time, regular military personnel).

The study is male only because females are excluded from the RCT of military personnel undergoing hip joint rehabilitation due to gender specific adaptations to strength exercises being a confounder for training adaptations in interventionist research [1]. Therefore, as this case-control study is nested in the RCT, it is not possible to include females.

\section{Selection and recruitment of cases}

Case participants are recruited from patients attending a Multidisciplinary Injury Assessment Clinic (MIAC) at DMRC who will be assessed for eligibility in accordance with the study inclusion/exclusion criteria. Potential participants will undergo a comprehensive musculoskeletal examination by a specialist rehabilitation consultant and experienced musculoskeletal physiotherapist.

Patients referred to DMRC with hip pain will be sent an information sheet prior to attending the MIAC detailing the purpose and nature of the study. The requirement to complete a study questionnaire will be highlighted. Following the MIAC, eligible patients will be provided with sufficient time (minimum 24-hours) to ask questions and consider factors surrounding their potential participation. Patients will then be invited to participate in the study and complete an informed consent form.

\section{Selection and recruitment of controls}

To avoid any potential bias control participants will 
Table 1: Content of method inventory and outcome parameters in Military Physical Loading Questionnaire (MPLQ).

\begin{tabular}{|c|c|c|c|}
\hline Parameter & Intention & Outcome & Source† \\
\hline Sociodemographic factors & $\begin{array}{l}\text { Matching variables and basic } \\
\text { description }\end{array}$ & Distribution & [32 and own development] \\
\hline Pre-entry activity \& exercise & Possible confounding factors & Cumulative effects & [32] \\
\hline Musculoskeletal symptoms \& injury history & Possible confounding factors & Health status & [32 and own development] \\
\hline $\begin{array}{l}\text { Frequency \& duration of military physical } \\
\text { tasks }\end{array}$ & Occupational exposure & Cumulative effects & $\begin{array}{l}{[2,16,28,34, \text { and own }} \\
\text { development] }\end{array}$ \\
\hline $\begin{array}{l}\text { Frequency \& duration of physical tasks - } \\
\text { operational deployment }\end{array}$ & $\begin{array}{l}\text { Occupational exposure on } \\
\text { operational deployment }\end{array}$ & Cumulative effects & $\begin{array}{l}\text { [2,16,28,34 and own } \\
\text { development] }\end{array}$ \\
\hline Frequency \& duration of sports participation & Possible confounding factors & Cumulative effects & [2,34 and own development] \\
\hline Alcohol, smoking, disease assessment & Possible confounding factors & $\begin{array}{l}\text { Cumulative effects \& } \\
\text { health status }\end{array}$ & $\begin{array}{l}\text { [31,34 and own } \\
\text { development] }\end{array}$ \\
\hline
\end{tabular}

$\dagger[2,16,28,32,34]$ cite research articles describing different studies and results.

be randomly selected from serving members of the UK Armed Forces. A dedicated MOD health statistician will identify control participants from existing data sources. After the case participant questionnaires have been reviewed for completeness and validity of data, the independent statistician will be instructed as to which controls are needed in order to match for age (year of birth), with each case. An information sheet explaining the purpose of the study and consent form will be sent to potential control participants via their secure MOD e-mail account at this time. In order to exclude those with current symptoms or undiagnosed hip disorders, potential controls will be contacted via telephone by a civilian project administrator and asked a series of screening questions to establish eligibility against the study inclusion and exclusion criteria.

\section{Data Collection Instrumentation}

\section{Standardised questionnaire}

A Military Physical Loading Questionnaire (MPLQ) examining physical activity levels and occupational mechanical loading prior to and during military service will be administered to each participant. The items included in this questionnaire are adapted from previous validated instruments used in population-based studies of lifestyle [32], occupational demands and hip OA $[2,14,16,17,23,28,29]$ (Table 1). The structured questionnaire contains sections enquiring about sociodemographic factors, physical characteristics, pre-enlistment activity levels, musculoskeletal symptoms, injury history, occupational physical loading, sports participation, and lifestyle factors including smoking and alcohol consumption. In order to further assess the validity of item responses in a military population, a pilot validation study will be undertaken with a representative sample of healthy military staff at DMRC Headley Court. The MPLQ employs 'skip logic' that allow participants to follow individualized paths through the questionnaire, moving forward based on responses to previous questions, thereby reducing the time taken to complete the questionnaire [33]. A copy of the MPLQ is available as an online additional file. This questionnaire is completed by all participants in the study.

\section{Measurement of pre-entry activity and exercise}

Pre-entry activity levels were incorporated into the MPLQ using items from the Military Pre-Training Questionnaire (MPQ) [32]. The items included in the MPLQ are designed to investigate specific aspects of the participants' pre-training exercise behavior. Selected questions have demonstrated criterion validity, and have been shown to predict injury risk in military recruits [34]. The MPQ pre-entry physical activity items have demonstrated excellent test-retest reliability [32].

\section{Measurement of occupational physical demands}

History of exposure to occupational physical demands is measured from the point of enlistment. Participants are asked about each job/posting/draft held for one year or longer up to a maximum of eight postings. Job number one describes the combined period of initial phase 1 and phase 2 military training. For each job, information is collected on age and rank at starting and finishing the job and if it involved each of 18 physical demands (section 4 - MPLQ additional file). The frequency of each physical task is rated on a 5-point scale, with 0 $=$ never and $4=$ very often. Participants are classified as "exposed" to the indicated task during each job if they performed that task "often" or "very often" during an average week, and non-exposed if they respond "never", "not very often" or "sometimes". This method of recording occupational physical demands has been used previously in community based hip pain studies and its construct validity demonstrated $[2,28,35]$.

\section{Measurement of occupational physical demands- deployed operations}

Participants will also be asked about performance of 18 physical tasks during their time (total summed months) spent on deployed military operations. Participants will provide information on the average number of hours in each 12-hour day (none, 0-1, 2-4, 5-7, 8+ hours) performing each of the 18 operational tasks (section 5 - MPLQ additional file). Participation in each specific task is calculated by taking the product of duration (total days on operations) multiplied by the self-report- 
ed length of participation each day (average number of hours). Output data will yield information used to asses if exposure to physical loading on operational deployments presents an additional risk for developing hip pain compared to other periods during a military career.

\section{Measurement of sports participation}

The questionnaire includes further items enquiring about lifetime participation in sport and recreational activities. Participants will be provided a list of $40 \mathrm{com}$ mon sports to which they can add additional activities. Participants will be asked to indicate the age they started/stopped the activity and for how long, on average, it was/is carried out each week; three response options are provided (less than 1 hour, 1-4 hours, more than 4-hours). This approach has emerged as a useful and reliable method for evaluating long-term participation in sport and recreational activity [33]. Pre-entry activity/exercise and sports participation could confound the relationship between occupational hip loading and hip pain and will be assessed as confounding factors in this study.

\section{Exposure assessment}

Person hours of exposure to occupational physical loading (section 4, additional file) will be calculated for each task by establishing the length of time spent carrying out the activity in years. To estimate incidence, 1-year of exposure is defined as every year of military service completed from point of enlistment (entry) to the point of onset of hip symptoms. Participants are classified as exposed if they rate their frequency of performing each task as "often" or "very often" in jobs/postings of 1-year. For each task exposure, three subclasses are defined on the basis of the distribution of exposure in the referent group (1) The $25 \%$ with the lowest exposure (low exposure group), (2) The $25 \%$ with the highest exposure (high exposure group), and (3) The $50 \%$ in-between groups 1 and 2 (medium exposure group).

Person hours of exposure to operational deployment physical loading (section 5 , additional file) will be calculated for each task by establishing the length of time spent carrying out the activity in months (total time on operations), converting into days, and multiplying by the hourly frequency of the activity using the following values: $0-1$ hours per day $=0.5$ hours; $2-4$ hours per day $=3$ hours; $5-7$ hours per day $=6$ hours; $8+$ hours per day $=9$ hours. For analysis, the cumulative duration of each operational physical task is categorized into high exposure, medium exposure and low exposure groups.

Person hours of sports participation exposure (section 6, additional file) will be calculated for each sport by establishing the length of time spent carrying out the activity in years (age stopped minus age started), converting years into weeks and multiplying by the hourly frequency of the activity using the following values: < 1 hour per week $=0.5$ hours; 1 - 4 hours per week $=2.5$ hours; $>4$ hours per week $=6$ hours. Three exposure categories are derived in an analogous manner to the occupational and operational physical loading exposure categories described above.

Body Mass Index (BMI) will be calculated as weight (in kilograms) divided by height 2 (in meters). Change in $\mathrm{BMI}$ is calculated as current BMI minus BMI on enlistment into the military. Based on current smoking behavior, participants are categorised into three groups: current smokers; previous but not current smokers; non-smokers.

\section{Questionnaire reliability}

The study questionnaire uses items adapted from existing validated instruments (Table 1 ). The test-retest reliability and internal consistency of the study questionnaire in a military population will be assessed with a sample of healthy male military permanent staff $(n=$ 50) from DMRC Headley Court. The inclusion/exclusion criteria and screening procedures will be the same for this sub-sample as those described for the control participants in the main study. Through necessity this element of the research programmer will be conducted as a pragmatic pilot study using a convenience sample. As a tri-service unit with a diverse mix of military branch, trades, rank and experience, DMRC provides access to a representative sample of serving UK military personnel.

Potential participants will be notified and recruited using publicity posters, and announcements on the organizational intranet webpage. Eligible volunteers will be provided with the study information sheet and consent form. Upon receipt of the signed consent form the questionnaire will be administered on two separate occasions with a time span of 4-weeks between questionnaire completions. The 4-week 'washout' period balances the need to minimize bias by reducing the tendency of respondents to recall previous responses, with the requirement to avoid a potential change in the construct being measured.

\section{Sample Size}

There is no published data describing the effects of occupational physical loading as a risk factor for non-arthritic hip pain in a young active cohort. Consequently, a power evaluation is difficult as methodological and situational differences do not allow direct determination of an appropriate sample size. The proposed sample size of 500 is therefore limited by practical considerations rather than a formal power calculation. This is based on the number of participants recruited into the phase 1 RCT who will comprise the case participants $(n=100)$, and is the estimated number of cases who can be recruited within the timescale of the study. A 4:1 matching ratio will increase the overall power of the study without the time dependent requirement to recruit additional case participants. This data will be used to inform a subsequent sample-size calculation for future studies. 


\section{Data Collection}

\section{Case participants}

Initial identification of potential case participants will occur during the consultant led outpatient MIAC at
DMRC Headley Court. Patients attending the MIAC with clinical indicators of non-arthritic, intra-articular hip pain who meet the inclusion/exclusion criteria will then be invited to participate in the study. Once eligibility has been confirmed, patients will be provided with a full

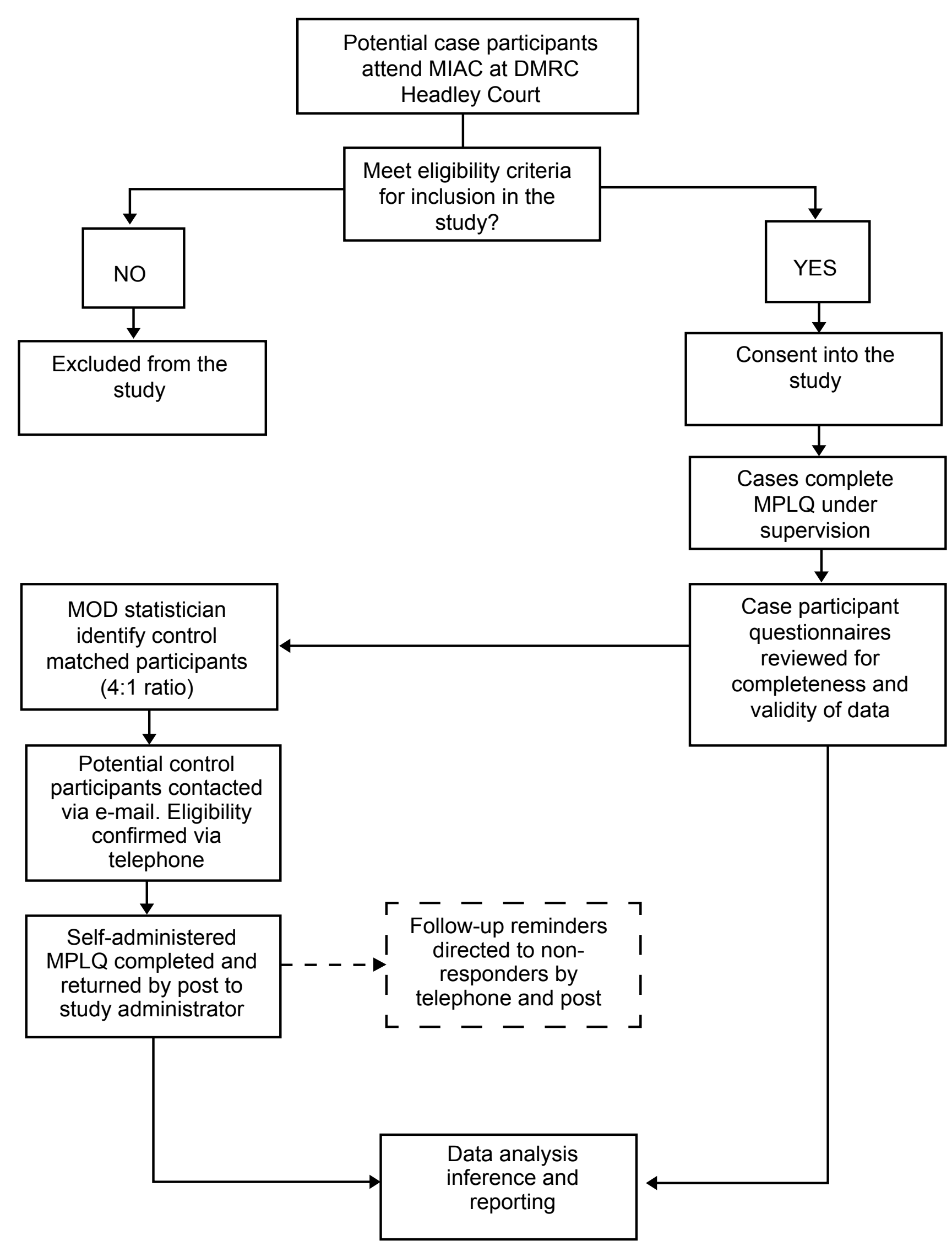

Figure 1: Schematic representation of study design, participant involvement and data collection process. 
description of the study by a member of the research team. A verbal explanation of the trial including clarification of the patient information sheet will be provided. Participants will be provided with a copy of the study questionnaire when written informed consent has been provided. Administration of the study questionnaire is performed on a single occasion, in a quiet environment at DMRC. The questionnaire will be completed under the supervision of a member of the research team, and participants will be encouraged to seek advice on items they do not fully understand.

\section{Control participants}

A dedicated MOD health statistician will identify control participants from existing data sources matched for age (date of birth). Control participants will be randomly selected and recruited from a sample of currently serving members of the UK Armed Forces population. Potential control participants will initially be approached via their secure professional military e-mail account. The initial e-mail will provide a general explanation of the research, a covering letter from the research team, an information sheet explaining the purpose of the study, and a consent form. Eligible control participants will be provided with the contact details of the chief investigator and study administrator should they require further information or wish to ask any questions prior to providing written informed consent. By completing the consent form, participants will be volunteering to 'opt in' to the study. When an informed written consent form has been returned, each control participant will be sent a hard-copy of the study questionnaire with instructions for completion and a self-addressed return envelope. Participants will be advised and encouraged to seek advice from the chief investigator for clarification on questionnaire items they do not fully understand. This query can be via telephone or secure UK Ministry of Defense e-mail and this facility is stressed in the participant information sheet. Whilst this does not replicate the direct 'on-site' personal communication enjoyed by case participants, it does provide the opportunity for control participants to seek all the clarification they require on any item responses (Figure 1).

\section{Statistical analysis}

Associations of hip pain with physical load will be examined with two sets of occupational variables; (a) Lifetime exposure to 18 occupational physical tasks and (b) Lifetime exposure to 18 operational deployment physical tasks. Associations of hip pain with lifetime sports participation will also be analyzed. For analysis, the cumulative duration of each occupational and operational physical task is categorized into three groups: (a) Low-Exposure; (b) Medium exposure; (c) High exposure.

Descriptive statistics will be performed to characterize the study sample; continuous variables will be summarized using means and standard deviations. Data assumptions will be checked for normality prior to analysis. The standardized residuals plot will be checked for outliers. Data will be checked for normality, linearity and homoscedasticity with visual inspection of the residual scatter plots. Conditional logistic regression will be used, and associations summarized as Odds Ratios (OR) with 95\% Confidence Intervals (Cl) to estimate the relationship between occupational/operational physical demands and hip pain between high and medium-exposure groups, compared with the low-exposure group. The odds ratio is interpreted as an estimate of the incidence rate ratio because the design is that of a population-based case-control study [36]. To identify those occupational/operational tasks independently associated with hip pain, a stepwise multivariate logistic regression analysis will be undertaken comprising all factors found to be significantly associated with hip pain in the univariate analysis. This will adjust for the effects of confounding between variables. Statistical significance of interactions will be tested with the likelihood ratio test and expressed as exact $p$-values. Statistical significance is taken when $P<0.05$.

The test-retest reliability of the MPLQ will be assessed by calculating Intraclass Correlation Coefficients (ICC) and $95 \% \mathrm{Cl}$ based on a one-way ANOVA model. Strength of agreement between test-retest responses for both categorical and continuous variables is characterized according to the ratings of Landis \& Koch [37]: poor $=0-0.2$, fair $=0.2-0.4$, moderate $=0.4-0.6$, substantial $=0.6-0.8$ and almost perfect $=0.8-1.0$. For index measures related to the same construct, internal consistency is assessed after the first administration by calculating Cronbach's alpha $(\alpha)$ coefficients. Internal consistency is deemed acceptable if $\alpha$ is $>0.7$ [32]. The statistical analysis procedure will be discussed, reviewed and co-worked with statisticians at the Department for Health University of Bath, and School of Clinical Sciences/School of Oral \& Dental Sciences at Bristol University.

\section{Content of method inventory/MPLQ}

The MPLQ used in the study is designed partly from published instruments (Table 1 ).

\section{Data storage and quality assurance}

All original documents and information obtained in the conduct of this study will be treated as confidential and participant anonymity will be ensured throughout the study period. Electronically stored data will be identified by a password-protected participant ID code unique to the study. We will transcribe data from paper forms directly into a bespoke study relational database (Concentrica Ltd, Cambridge, UK) based on servers in the Academic Department of Military Rehabilitation (ADMR), DMRC and the Department for Health, University of Bath. To minimize transcription error, we will use manual double data entry. $R C, J B, A W$ and $A B$ will have access to the final trial dataset. 


\section{Risks and adverse events}

All researchers involved in the conduct and supervision of this study will receive extensive training in all aspects of the administration of the trial protocol. As far as we are aware no undesirable effects will arise as a consequence of completing the study questionnaire and there are no specific risks associated with this study. In the unlikely event this study is prematurely terminated for any reason, the MOD research ethics committee will be informed and provided with the justification for this action.

\section{Publication policy}

We will submit the results of our study for publication in a suitable journal regardless of the outcomes. The chief investigator will take responsibility for producing draft report manuscripts and all co-investigators will review and approve the study results prior to submission for publication. Authorship of all manuscripts and presentations will comply with the ICMJE "Uniform requirements for Manuscripts Submitted to biomedical Journals" [38].

\section{Discussion}

This case-control study will provide the first estimates of the risks associated with occupational factors and the onset of non-arthritic hip pain in UK military personnel. We anticipate this research will establish if cumulative, life-long physical loading represents a true occupational hazard in military personnel. If aspects of specific occupational activities and tasks contribute to the occurrence of hip pain it will inform future military specific prevention strategies. The research may also provide a greater understanding of the pathogenesis of hip pain not only in the military context but occupational health related to young adults exposed to high physical workload.

Prospective studies that follow each subject with repeated exposures over a prolonged period are labor intensive and expensive. For that reason we will use retrospective questionnaire assessment of occupational exposure. The weakness of retrospective participant self-recall of historical events is well recognized as a potential source of bias in epidemiological research. A degree of misclassification is probably present in all studies using retrospective exposure assessment and the possibility of participants under or over-estimating exposure will be highlighted as a limitation and potential confounder in our study. Furthermore, we cannot discount the possibility of experiencing selection bias in our study. By including case participants nested in a RCT we are confident our cases will have clinically diagnosed hip pathology. However, it is possible those with more physically demanding trades are disproportionately affected by a given level of symptoms and more likely to seek treatment. It is therefore possible associations with occupational activity levels could be spuriously exagger- ated in our cases [39]. The use of a well-defined military population may limit the generalizability of our results to other studies.

The study has some methodological strengths. Our questionnaire uses prompts surrounding specific jobs, operational deployments and sports participation to stimulate recall of activities performed towards the onset of hip pain. A recent review showed that self-reports on work-related physical factors are quite reliable as people can more easily recall issues such as the number of years in a particular job [40]. We will use clinically and epidemiologically validated methods for assessing intra-articular hip pain, occupational exposure and physical activity. Results will be reported in accordance with the Reporting of Observational Studies in Epidemiology (STROBE) Statement [41] and study completion is anticipated by early 2020 .

\section{Acknowledgements}

Thanks to the staff and patients on the Centre for Lower Limb Rehabilitation at DMRC for their support in delivering this study, particularly Mr Stuart Phazey. Thanks to the Medical Officers at selected UK military feeder units for identifying and referring the research participants. Thanks to the MILO study administrator Mr. Toby Okoye. Thanks to Richard Dixon, Tony Jones, Christine Poole and staff at 22 Training Group (HQ Air) Media Graphics department for producing supporting literature for the study.

\section{Ethical Statement}

The study has been planned and conducted in accordance with the UK Ministry of Defense (MOD) policy for research using human participants and the Helsinki Declaration 2013 [42]. The study protocol and its amendment were approved by the MOD research ethics committee (approval code 651 dated 01 Aug 2015).

Potential participants will be provided with detailed written information about the voluntary nature of the study. The information will outline the benefits of taking part in the study whilst accurately reflecting the absence of any risk associated with completing the study questionnaire. The right for the participant to refuse consent without providing reasons and without prejudice will be emphasized, and volunteers will be free to withdraw from the study at any time without prejudicing any further treatment. The process of obtaining written consent will be clearly documented in the patient's medical notes (cases) and investigator site file.

\section{Additional Material}

Additional file: Military Physical Loading Questionnaire (MPLQ).

\section{Competing Interests}

The authors declare they have no competing interests. 


\section{Funding}

This study is funded by the Arthritis Research UK Centre for Sport, Exercise and Osteoarthritis (Grant reference 20194).

\section{References}

1. Bennell KL, Egerton $\mathrm{T}$, Pua $\mathrm{YH}$, Abbott $\mathrm{JH}$, Sims $\mathrm{K}$, et al. (2010) Efficacy of a multimodal physiotherapy treatment program for hip osteoarthritis: a randomised placebo-controlled trial protocol. BMC Musculoskelet Disord 11: 238.

2. D Pope, I Hunt, F Birrell, A Silman, G Macfarlane (2003) Hip pain onset in relation to cumulative workplace and leisure time mechanical load: a population based case-control study. Ann Rheum Dis 62: 322-326.

3. Fransen M, McConnell S, Hernandez-Molina G, Reichenbach $S$ (2009) Exercise for osteoarthritis of the hip. Cochrane Database Syst Rev.

4. Pinto D, Robertson CR, Hansen P, Abbott JH (2012) Cost-effectiveness of nonpharmacologic, nonsurgical interventions for hip and/or knee osteoarthritis: Systematic review. Value Health 15: 1-12.

5. Eneski K, Harris-Hayes M, White DM, Cibulka MT, Woehrle J, et al. (2014) Nonarthritic hip joint pain. J Orthop Sports Phys Ther 44: 1-32.

6. Griffin DR, Dickenson EJ, O'Donnel JO, Agricola R, Awan T, et al. (2016) The Warwick agreement on femoroacetabular impingement syndrome (FAl syndrome): an international consensus statement. Br J Sports Med 50: 1169-1176.

7. Wright AA, Hegedus EJ, Taylor JB, Dischiavi SL, Stubbs AJ (2016) Non-operative management of femoroacetabular impingement: a prospective, randomized controlled clinical trial pilot study. J Sci Med Sport 19: 716-721.

8. Fairley J, Wang Y, Teichtahl AJ, Seneviwickrama M, Wluka $A E$, et al. (2016) Management options for femoroacetabular impingement: a systematic review of symptom and structural outcomes. Osteoarthritis Cartilage 24: 1682-1696.

9. Frank JM, Harris JD, Erickson BJ, Slikker W, Bush-Joseph CA, et al. (2015) Prevalence of femoroacetabular impingement imaging findings in asymptomatic volunteers: a systematic review. Arthroscopy 31: 1199-1204.

10. Agricola R, Weinans $H$ (2015) What is femoroacetabular impingement?. Br J Sports Med 50.

11. Agricola R, Heijboer MP, Bierma-Zeinstra SM, Verhaar JA, Weinans $\mathrm{H}$, et al. (2013) Cam impingement causes osteoarthritis of the hip: a nationwide prospective cohort study (CHECK). Ann Rheum Dis 72: 918-923.

12. Agricola R, Waarsing JH, Arden NK, Carr AJ, Bierma-Zeinstra SM, et al. (2013) Cam impingement of the hip: a risk factor for hip osteoarthritis. Nat Rev Rheumatol 9: 630-634.

13. Cheng Y, Macera CA, Davies DR, Ainsworth BE, Troped PJ, et al. (2000) Physical activity and self-reported, physician-diagnosed osteoarthritis: is physical activity a risk factor? J Clin Epidemiol 53: 315-322.

14. Kaila-Kangas L, Arokoski J, Impivaara O, Viikari-Juntra E, Leino-Arjas P, et al. (2011) Associations of hip osteoarthritis with history of recurrent exposure to manual handling of loads over $20 \mathrm{Kg}$ and work participation: a population-based study of men and women. Occup Environ Med 68: 734-738.

15. Juhakoski R, Heliovaara M, Impivaara O, Kroger H, Knekt $P$, et al. (2009) Risk factors for the development of hip osteoarthritis: a population-based prospective study. Rheumatology 48: 83-87.
16. Yoshimura N, Sasaki S, Iwasaki K, Danjoh S, Kinoshita H, et al. (2000) Occupational lifting is associated with hip osteoarthiritis: A Japanese case-control study. J Rheumatol 27: 434-440.

17. Vignon E, Valat JP, Rossignol M, Avouac B, Rozenberg S, et al. (2006) Osteoarthritis of the knee and hip and activity: a systematic international review and synthesis (OASIS). Joint Bone Spine 73: 442-455.

18. Sulsky SI, Carlton L, Bochmann F, Ellegast R, Glitsch U, et al. (2012) Epidemiological evidence for work load as a risk factor for osteoarthritis of the hip: A systematic review. PLoS One 7: e31521.

19. Fransen M, Agaliotas M, Bridgett L, Mackay MG (2011) Hip and knee pain: Role of occupational factors. Best Pract Res Clin Rheumatol 25: 81-101.

20. Kopec JA, Rahman MM, Berholt JM, Le Petit C, Aghanjananian J, et al. (2007) Descriptive epidemiology of osteoarthritis in British Columbia, Canada. J Rheumatol 34: 386-393.

21. Ochoa LM, Dawson L, Patzkowski MD, Hsu JR (2010) Radiographic prevalence of femoroacetabular impingement in a young population with hip complaints is high. Clin Orthop Relat Res 468: 2710-2714.

22. Scher DL, Belmont PJ, Mountcastle S, Owens BD (2009) The incidence of primary hip osteoarthritis in active duty US military service members. Arthritis Rheum 61: 468-475.

23. Roach KE, Persky V, Miles T, Budiman-Mak E (1994) Biomechanical aspects of occupation and osteoarthritis of the hip: a case-control study. J Rheumatol 21: 2334-2340.

24. Cameron KL, Hsiao MS, Owens BD, Burks R, Svoboda SJ (2011) Incidence of physician-diagnosed osteoarthritis among active duty United States military service members. Arthritis Rheum 63: 2974-2982.

25. Jorgensen AY, Waterman BR, Hsiao MS, Belmont PJ (2013) Functional outcomes of hip arthroplasty in active duty military service members. J Surg Orthop Adv 22: 16-22.

26. Jensen LK (2008) Hip osteoarthritis: influence of work with heavy lifting, climbing stairs, or ladders, or combining kneeling/squatting with heavy lifting. Occup Environ Med 65: 6-19.

27. Hack K, Di Primio G, Rakhra K, Beaule PE (2010) Prevalence of cam-type femoroacetabular impingement morphology in asymptomatic volunteers. J Bone Joint Surg Am 92: 2436-2444.

28. P Croft, D Coggon, M Cruddas, C Cooper (1992) Osteoarthritis of the hip: an occupational disease in farmers. BMJ 304: 1269-1272.

29. Rubak TS, Svendsen SW, Soballe K, Frost P (2014) Total hip replacement due to primary osteoarthritis in relation to cumulative occupational exposures and lifestyle factors: a nationwide nested case-control study. Arthritis Care Res 66: 1496-1505.

30. Russell J Coppack, James L Bilzon, Andrew K Wills, lan M McCurdie, Laura Partridge, et al. (2016) A comparison of multidisciplinary team residential rehabilitation with conventional outpatient care for the treatment of non-arthritic intra-articular hip pain in UK Military personnel - a protocol for a randomised controlled trial. BMC Musculoskelet Disord 17: 459 .

31. Hunt D, Prather H, Harris Hayes M, Clohisy JC (2012) Clinical outcomes analysis of conservative and surgical treatment of patients with clinical indications of prearthritic, intra-articular hip disorders. PM R 4: 479-487. 
32. Robinson M, Stokes K, Bilzon J, Standage M, Brown $P$, et al. (2010) Test-retest reliability of the military pre-training questionnaire. Occup Med 60: 476-483.

33. Ratzlaff CR, Steininger G, Doerfling P, Koehoorn M, Cibere $J$, et al. (2011) Influence of lifetime hip joint force on the risk of self-reported hip osteoarthritis: a community-based cohort study. Osteoarthritis Cartilage 19: 389-398.

34. Knapik JJ, Sharp MA, Canham-Chervak M, Hauret K, Patton JF, et al. (2001) Risk factors for training-related injuries among men and women in basic combat training. Med Sci Sports Exerc 33: 946-954.

35. Allen KD, Chen JC, Callahan LF, Golightly YM, Helmick CG, et al. (2010) Associations of occupational tasks with knee and hip osteoarthritis: the Johnson county osteoarthritis project. J Rheumatol 37: 842-850.

36. Vingard E, Alfredsson L, Malchau H (1998) Osteoarthritis of the hip in women and its relationship to physical load from sports activities. Am J Sports Med 26: 78-82.
37. Landis JR, Koch GG (1977) The measurement of observer agreement for categorical data. Biometrics 33: 159-174.

38. (2010) Uniform requirements for manuscripts submitted to biomedical journals: writing and editing for biomedical publication. J Pharmacol Pharmacother 1: 42-58.

39. Coggon D, Kellingray S, Inskip H, Croft P, Campbell L, et al. (1998) Osteoarthritis of the hip and occupational lifting. Am J Epidemiol 147: 523-528.

40. Stock SR, Fernandes R, Delisle A, Vezina N (2005) Reproducibility and validity of workers self-reports of physical work demands. Scand J Work Environ Health 31: 409-437.

41. von Elm E, Altman DG, Egger M, Pocock SJ, Gotzsche PC, et al. (2008) The strengthening the reporting of observational studies in epidemiology (STROBE) statement: guidelines for reporting observational studies. J Clin Epidemiol 61: 344-349.

42. (1994) Recommendations Guiding Medical Doctors in Biomedical Research Involving Human Subjects. World Medical Association Declaration of Helsinki. J Med Liban 42: 88-89. 